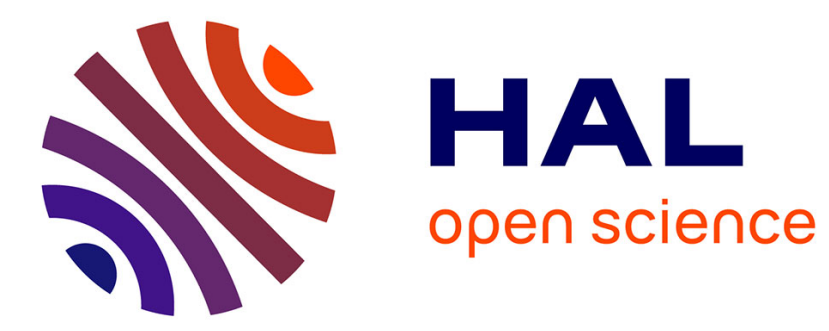

\title{
Leptospira and paramyxovirus infection dynamics in a bat maternity enlightens pathogen maintenance in wildlife
}

Muriel Dietrich, David A Wilkinson, Aude Benlali, Erwan Lagadec, Beza Ramasindrazana, Koussay Dellagi, Pablo Tortosa

\section{To cite this version:}

Muriel Dietrich, David A Wilkinson, Aude Benlali, Erwan Lagadec, Beza Ramasindrazana, et al.. Leptospira and paramyxovirus infection dynamics in a bat maternity enlightens pathogen maintenance in wildlife. Environmental Microbiology, 2015, 17 (11), pp.4280-4289. 10.1111/1462-2920.12766 . hal-01145007

\section{HAL Id: hal-01145007 \\ https://hal.science/hal-01145007}

Submitted on 24 Apr 2015

HAL is a multi-disciplinary open access archive for the deposit and dissemination of scientific research documents, whether they are published or not. The documents may come from teaching and research institutions in France or abroad, or from public or private research centers.
L'archive ouverte pluridisciplinaire HAL, est destinée au dépôt et à la diffusion de documents scientifiques de niveau recherche, publiés ou non, émanant des établissements d'enseignement et de recherche français ou étrangers, des laboratoires publics ou privés. 


\section{Leptospira and Paramyxovirus infection dynamics in a bat maternity}

2 enlightens pathogen maintenance in wildlife

3

4 Muriel Dietrich ${ }^{1,2}$, David A. Wilkinson ${ }^{1,2}$, Aude Benlali ${ }^{1,2}$, Erwan Lagadec $^{1,3}$, Beza

5 Ramasindrazana ${ }^{1,2,3}$, Koussay Dellagi ${ }^{1,3}$ and Pablo Tortosa ${ }^{1,2,4}$

6

$7{ }^{1}$ Centre de Recherche et de Veille sur les maladies émergentes dans l'Océan Indien, Sainte

8 Clotilde, Réunion Island, France ; ${ }^{2}$ Université de La Réunion, Sainte Clotilde, Réunion

9 Island, France ; ${ }^{3}$ Institut de Recherche pour le Développement, Sainte Clotilde, Réunion

10 Island, France ; ${ }^{4}$ Joint chair Centre National de la Recherche Scientifique - Université de La

11 Réunion, Sainte Clotilde, Réunion Island, France

12

13 Corresponding author: Muriel Dietrich, CRVOI, GIP CYROI, 2 rue Maxime Rivière, BP 14 80005, 97490 Sainte Clotilde, La Réunion, France. Tel: +262 2629388 20, Email:

15 muriel.dietrich@gmail.com

16

17 Short title: Dual infection dynamics in a bat maternity 


\section{Summary}

19 Bats are reservoirs for several zoonotic pathogens of medical importance; however infection dynamics of pathogens in wild bat populations remain poorly understood. Here, we examine

21 the influence of host crowding and population age structure on pathogen transmission and 22 diversity in bat populations. Focusing on two pathogen taxa of medical importance, 23 Leptospira bacteria and paramyxoviruses, we monitored host population and pathogen 24 shedding dynamics within a maternity colony of the tropical bat species Mormopterus 25 francoismoutoui, endemic to Réunion Island. Our data reveal astonishingly similar infection dynamics for Leptospira and paramyxoviruses, with infection peaks during late-pregnancy and two-months after the initial birth pulse. Furthermore, though co-infection occurs frequently during the peaks of transmission, the patterns do not suggest any interaction between the two pathogens. Partial sequencing reveals a unique bat-specific Leptospira strain contrasting with the co-circulation of four separate Paramyxovirus lineages along the whole

31 breeding period. Patterns of infection highlight the importance of host crowding in pathogen transmission and suggest that most bats developed immune response and stop excreting pathogens. Our results support that bat maternity colonies may represent hotspots of transmission for bacterial and viral infectious agents and highlight how seasonality can be an important determinant of host-parasite interactions and disease emergence. 


\section{Introduction}

Seasonal changes can have important effects on infectious disease dynamics (Altizer et al., 2006). Of the many driving factors of infection that demonstrate seasonal cycles, annual reproduction has been identified as having a significant impact on the dynamics of various host-parasite interactions, particularly in animal species that are gregarious during the reproductive season (Altizer et al., 2006). Indeed, during this period, aggregation within colony, increased metabolic activity and the highly synchronized birth of newborns (i.e. birth pulse) lead to increased numbers of susceptible hosts and hence boost the potential for transmission of infectious agents (e.g. Hosseini et al. 2004). Investigating the infection dynamics during host breeding seasons may therefore help to better identify major mechanisms of pathogen maintenance within wild animal populations.

Bats play important ecological roles in prey and predator cycles, arthropod suppression, seed dispersal, pollination, as well as nutrient distribution and recycling (Kunz et al., 2011). Additionally, bats have been identified as natural reservoirs of many microbial agents, including pathogens of threat to human health (Wibbelt et al., 2010). Their ability to enter torpor or hibernation, the large sizes of their gregarious groups and possibly some peculiarities in their immune system, have been suggested to facilitate maintenance of infections in these unique long-lived flying mammals (Calisher et al., 2006). The role of seasonal breeding in bat infection dynamics has been the subject of several recent studies (Amengual et al., 2007; Turmelle et al., 2010; Drexler et al., 2011; Field et al., 2011; Mühldorfer et al., 2011), mostly focusing on viruses. For example, lyssaviruses and henipaviruses have been observed to exhibit robust and specific amplification in maternity colonies (Drexler et al., 2011; George et al., 2011). Furthermore, although multi-pathogen interactions have been shown to drive infection dynamics in other host-parasite models (e.g. Telfer et al. 2010), very few studies have considered the dynamics of multiple infections in 
natural bat populations (Drexler et al., 2011; Mühldorfer et al., 2011).

Leptospirosis is considered as the most common bacterial zoonosis in the world and is caused by pathogenic spirochetes of the genus Leptospira (Adler and de la Peña Moctezuma, 2010). The incidence of human leptospirosis is high in tropical islands, particularly in the Indian Ocean Islands where some of the highest human incidences have been reported to date (Pappas et al., 2008). Chronic carrier animals are characterized by persistent leptospire colonization of renal tubules, which are then shed for months or years in urine and contaminate the environment. Human infection occurs either directly from contact with animal reservoirs or indirectly from contaminated soil or water (Adler and de la Peña Moctezuma, 2010). Rodents are classically recognized as the most significant reservoirs for the maintenance and dissemination of leptospires worldwide (Bharti et al., 2003). More recently, bats have also been identified as potential reservoirs of leptospires in a number of locations (e.g. Matthias et al. 2005; Tulsiani et al. 2011), with sometimes very high infection rates (Lagadec et al., 2012). Mathematical modeling has suggested seasonality of host reproduction as an important factor affecting Leptospira infection dynamics in rodent populations (Holt et al., 2006), however, no published information is currently available concerning Leptospira infection dynamics and maintenance in bat populations.

The Paramyxoviridae form a large virals family including the causative agents of many human and animal diseases, and some that have recently been linked to emerging and reemerging epidemics (e.g. Hendra and Nipah viruses; Aguilar \& Lee, 2011). Rodents and bats have been recently shown to host a broad spectrum of paramyxoviruses (Drexler et al., 2012; Wilkinson et al., 2012; Baker, Todd, et al., 2013). Phylogenetic reconstruction of host/virus associations suggests frequent spillover events between different hosts, with a predominance of host shifts from bats to other animal species (Drexler et al., 2012; Wilkinson et al., 2014), which has elsewhere been linked with the bat reproduction period (Plowright et al., 2008). For 
instance, Plowright et al. (2008) have identified reproduction as a key factor in the risk of Hendra virus infection in Australian fruit bats and especially in the emergence of these viruses in horse populations.

In this study, we investigated the dynamics of two pathogens of medical importance, the bacteria Leptospira and Paramyxoviruses, which have previously been described in insectivorous bats (Lagadec et al., 2012; Wilkinson et al., 2012). The tropical and endemic bat species Mormopterus francoismoutoui is the most abundant bat species on Réunion Island, and roosts in large colonies in a variety of sites such as bridges, houses, churches and caves. We chose to study the largest known cave-based maternity colony of these bats in order to (i) determine whether the infection rate and/or the genetic diversity of infectious agents are influenced by host crowding and the succession of different developmental stages (adults, newborns and juveniles) over the breeding season, and (ii) compare the temporal infection profiles of both infectious agents. We hypothesize that transmission is host-density dependent favored by colony-induced crowding and fueled by the input of immunologically naïve juveniles (Drexler et al., 2011). Results are expected to reveal specific characteristics of the ecology and evolution of Leptospira and paramyxoviruses and to highlight general patterns in pathogen maintenance in bat populations.

\section{Results}

\section{Bat colony dynamics}

The bat population studied was strictly composed of adults at the beginning of the survey in late November (Fig. 1a). A large number of newborns were observed in early January, together with the first juveniles ( $\sim 3$ weeks old), indicating that the birth pulse occurred from mid-December to mid-January. We estimated that the parturition period lasted up to midFebruary as the last observation of newborns was made on February $8^{\text {th }}$. 
Over all outings, a total of 2652 urine droplets were counted on the 16 plastic trays. The spatial distribution of the urine droplets suggested that bats use a main route for exiting the cave: those trays facing the center of the entrance were more exposed to bat passage than the ones placed on each side of the main flyway $\left(\chi_{15}^{2}=49.26, \mathrm{p}<0.001\right.$; model 1 in Table S3).

116 Trays lying directly underneath the main bat flyway (yellow-red in Fig. S1b) showed

117 statistically significant variations in the number of urine droplets collected over time $\left(\chi^{2}{ }_{7}=\right.$ 118 48.43, p < 0.001; Fig. $1 \mathrm{~b}$ and model 1 in Table S3). Indeed, at the first sampling session (i.e.

119 November $20^{\text {th }}$ ), the number of exiting adult bats reached a maximum: 46500 individuals as 120 estimated by the video. Following this maximum, the number of exiting bats declined 121 drastically to reach a minimum on January and remained very low for about three additional 122 weeks. This corresponds to the parturition period where females gave birth and thus remained 123 with newborns leading to this reduced pattern of exit. Then, the number of exiting bats 124 increased to reach a second peak on mid-February, coinciding with the first flights of juvenile 125 bats. Beyond that time point, the number of exiting bats declined progressively towards the 126 end of the season corresponding to the definitive emptying of the cave; the number of exiting 127 bats estimated by video analysis was still 13400 individuals at the end of the sampling period 128 (i.e. March $21^{\text {st }}$ ). In late May, a last visual inspection showed that the cave was unoccupied.

\section{Leptospira and paramyxovirus infection dynamics}

131 Altogether, 420 individual urine droplets were screened by PCR for Leptospira and 132 paramyxoviruses during the $2012-2013$ breeding season. The bacterial and viral excretion 133 positively identified individual bats as being currently infected with either of the two 134 infectious agents. Hereafter, 'infection rate' refers to the proportion of PCR-positive samples.

135 Both Leptospira and paramyxovirus infection rates varied significantly over the breeding 136 season (Leptospira: $\chi_{7}^{2}=25.209, \mathrm{p}<0.001$; paramyxovirus: $\chi^{2}{ }_{7}=59.879, \mathrm{p}<0.001$, models 2 
and 3 in Table S3) and revealed similar patterns of temporal variation (Fig. 1c). The relative

138 intensity of Leptospira excretion also showed significant variation over time, despite the large

139 variation in the $\mathrm{Ct}$ values $\left(\chi_{7}^{2}=148.06, \mathrm{p}=0.003\right.$, fig. $1 \mathrm{~d}$ and model 4 in Table S3). Overall,

140 infection dynamics were characterized by two peaks, both in the rates of infection (Fig. 1c)

141 and the intensity of Leptospira excretion (Fig. 1d). Notably, the first peak occurred at the

142 beginning of the breeding season, during the late-pregnancy period of females, with infection

143 rates $( \pm 95 \%$ confidence interval) reaching $45 \%( \pm 12 \%)$ and $61 \%( \pm 11 \%)$ for Leptospira and

144 paramyxovirus, respectively. Later, during the parturition period, both infection rates (as well

145 as the intensity of Leptospira excretion) decreased drastically down to a minimum of $6 \%$

$146( \pm 6 \%)$ and $4 \%( \pm 5 \%)$ for Leptospira and paramyxovirus respectively (Table S4). The second

147 infection peak (as well as the intensity of Leptospira excretion) occurred roughly two months

148 after the beginning of the parturition period. Towards the end of the season, infection rates

149 slightly decreased, with Leptospira and paramyxovirus detection rates reaching $21 \%( \pm 11 \%)$

150 and $31 \%( \pm 13 \%)$, respectively. Infection rates were also monitored at the start of the

151 following season in order to collect data on the early stages of colony formation. Infection

152 rates were $28 \%( \pm 16 \%)$ and $21 \%( \pm 16 \%)$ on October $18^{\text {th }} 2013$ for Leptospira and

153 paramyxovirus respectively, and increased within 2 weeks, to reach $62 \%( \pm 18 \%)$ and $52 \%$

$154( \pm 18 \%)$ respectively (Table S4).

155 Our data showed that bats were frequently co-infected (Fig. 2a). Statistically, the 156 presence of one infectious agent was strongly correlated to the presence of the other agent $\left(\chi_{1}^{2}\right.$ $157=22.170, \mathrm{p}<0.001$, models 2 and 3 in Table S3). Moreover, the rate of co-infection 158 significantly varied over the breeding season $\left(\chi_{7}^{2}=41.761, \mathrm{p}<0.001\right)$ and followed the same 159 dynamics as mono-infections (Fig. $2 \mathrm{~b}$ and model 5 in Table S3). However, the expected 160 probability of being co-infected at random for each sampling date was not significantly 161 different from the observed values (G-test: $p>0.1$ for all tests; Fig. 2b). Finally, the intensity 
162 of Leptospira excretion was not affected by co-infection with paramyxovirus $\left(\chi_{1}^{2}=0.020, \mathrm{p}=\right.$ 163 0.957, model 4 in Table S3).

\section{Genetic diversity of Leptospira and paramyxovirus}

166 Phylogenetic analyses of Leptospira provided similar results for $\sec Y$ and $r r s 2$ genes (Fig. 3a

167 and Fig. S4 respectively). Both phylogenetic trees revealed the circulation of a single

168 haplotype in the maternity colony, closely related to Leptospira borgpetersenii. This

169 haplotype was distinct from those previously described in wild animals and humans in the

170 western Indian Ocean. In contrast, paramyxoviruses detected in the cave were much more

171 diverse as 4 different genetic groups were observed (Fig. 3b). The first group corresponds to

172 virus sequences associated with three different bat families of the western Indian Ocean

173 region (Molossidae, Miniopteridae and Verspertilionidae). The second group includes virus 174 sequences detected in the very closely related bat species $M$. acetabulosus (Mauritius) and $M$.

175 jugularis (Madagascar). Virus sequence in the third group were initially identified in kidney

176 tissue from a rat caught in Réunion Island near the studied cave (Wilkinson et al., 2014).

177 Finally, one sequence grouped with viruses largely associated with rats in the region (group

178 4). There was no evidence of any correlation between the occurrence of the different

179 Paramyxovirus genetic groups and sampling date $(p=0.467)$, suggesting that the 4 genetic

180 groups were likely co-circulating within the colony.

182 Discussion

183 Previous studies have demonstrated that dynamics of bat-hosted infectious agents exhibit a 184 strong seasonal pattern, with infection peaks being recorded during the breeding period 185 (Turmelle et al., 2010; Drexler et al., 2011). Here, using a non-invasive sampling strategy in a 186 natural maternity colony, we monitored the excretion of both a bacterial and a viral agent, 
namely Leptospira and paramyxovirus, by a tropical insectivorous bat species endemic to

188 Réunion Island. Our data provide evidence that infection rates express strong temporal 189 fluctuations within the time scale of the breeding season. We find that Leptospira and 190 paramyxoviruses display remarkably comparable infection dynamics, a feature suggestive of 191 synchronized host susceptibility and a common transmission route within the colony, i.e. 192 urine. Data from two successive breeding seasons showed epidemic bursts occurring during 193 colony formation and two-months after the birth pulse. These findings strongly suggest the 194 occurrence of a vigorous horizontal transmission within the maternity colony and support that 195 seasonal coloniality and synchronized bat behavior is a major determinant of infection 196 dynamics at the population level. Synchronized host susceptibility in females may have resulted from a transient 198 pregnancy-related depressed immunity. Increased susceptibility to infectious agents during 199 pregnancy has been demonstrated in different mammalian systems (e.g. Sheldon \& Verhulst 1996; Cattadori et al. 2005) and short-term trade-offs between immune activity and 201 reproduction have been suggested to explain such patterns. In the greater mouse-eared bat 202 (Myotis myotis), for instance, weaker cell-mediated responses and heavier ectoparasite 203 infestations have been observed in pregnant females compared to non-reproductive females 204 from the same roost (Christe et al., 2000). Our sampling design does not give access to the 205 immunological status of the population, and thus does not allow verification of this 206 hypothesis. However, such undulating patterns of immunomodulation and viral replication in 207 females during late-pregnancy have already been reported in other field studies investigating 208 Hendra or filo-viruses dynamics in frugivorous bats (e.g. Plowright et al. 2008; Breed et al. 209 2011).

210 Following the infection burst that occurs early-on in colony formation, a rapid and 211 highly synchronous decline in Leptospira and paramyxovirus excretion was observed, 
suggesting that a large majority of adult bats rapidly control the acute infection and that

213 excretion of infectious agents is only transitory. This pattern is most likely the consequence of

214 the synchronous development of a robust immune response in bats against the two infectious agents. Such an immune response would not only protect parturient females, but also newborns through the passive transfer of specific antibodies from mother to offspring as

217 demonstrated experimentally in frugivorous bat species (Baker, Suu-Ire, et al., 2013; Epstein 218 et al., 2013), and largely suggested by several field studies (Plowright et al., 2008; Breed et 219 al., 2011), including one carried out on insectivorous bats (Drexler et al., 2011). Though 220 transitory, the conferred passive protection may shape age related patterns of infection 221 (Boulinier and Staszewski, 2008) and has been suggested to last between one and six months 222 after maternal severing in insectivorous (Drexler et al., 2011) and frugivorous bats (Baker, 223 Suu-Ire, et al., 2013; Epstein et al., 2013), respectively. Thus, the influx of juveniles in the 224 group of susceptible bats is delayed until the disappearance of passively transferred maternal 225 antibodies. Indeed, the two-months delay observed before appearance of the second infection 226 peak is consistent with such waning of passively transferred maternal antibodies, as 227 previously suggested by a field study on Myotis bats (Drexler et al., 2011). In addition to an 228 immunization of the mothers, the emergence of uninfected juveniles may contribute to the 229 prevalence decrease measured in February. However, considering that females give birth to a 230 single pup, this decrease should be at maximum of 50\%, which is consistently lower than the 231 actual observed drop (i.e. xx\% for Leptospira and xx\% for paramyxovirus, respectively). 232 Thus we propose that the dramatic decrease in prevalence results from both immunization of 233 the mothers and the emergence of transiently immunized juveniles. Interestingly, some of the 234 expected positive relationship between host density and infection prevalence (Kallio et al., 235 2010) may be lacking at the end of the season when host density has increased by twice with 236 the birth of newborns. The number of immune females may explain why infection rates, and 
especially that of paramyxovirus, did not return to the peak levels measured at the beginning of the season. Further investigations addressing the serological status of pregnant mothers, newborns and juveniles towards Leptospira and paramyxovirus need to be carried out in order to properly address these major points.

Seasonality of reproduction might play a role in the evolution of infectious agents by causing alternating periods of potential mixing of different lineages with high transmission and population bottlenecks that simultaneously limit strain diversity and cause rapid genetic shifts (e.g. Ferguson et al., 2003). In the present study, we observed that one single haplotype of Leptospira co-circulated with multiple paramyxovirus lineages, though no apparent evolution of the viral diversity could be recorded over the timescale of our observations. Multiple circulating virus strains may affect within-host and within population dynamics by conferring cross-immunity (Hayman et al., 2013). Interestingly, we also found evidence of paramyxovirus sharing between bat and rodent populations, suggesting that host shift between rats and bats may contribute to the paramyxovirus diversity observed within the maternity colony.

The presence of multiple infectious agents within a host, i.e. co-infection, can critically impact infection dynamics (Telfer et al., 2010). Interactions may be synergistic or antagonistic and infectious agents may interact either directly or indirectly via 'bottom-up' (e.g. competition for shared host resources) or 'top-down' processes (e.g. immune-mediated competition or facilitation) (Pedersen and Fenton, 2007). Here, although bats were frequently co-infected with Leptospira and paramyxovirus, the observed rate of co-infection was not different from what is expected at random and we found that the presence of paramyxovirus infection had no effect on the intensity of Leptospira excretion. The possibility that the intensity of paramyxovirus infection could be positively influenced by Leptospira infection could not be tested with our experimental setup and, thus, cannot be ruled out. The large 
confidence intervals for pathogen prevalence and intensity of Leptospira infection preclude

263 any solid conclusion but our study suggest that co-infection most likely appears as the mere consequence of the temporal conjunction between the infection dynamics and did not result in any apparent interaction between the two pathogens.

In conclusion, our study has focused on infection dynamics within the time frame of the bat breeding season. Although it is difficult to distinguish between episodic shedding from persistently infected bats and transient epidemics (Plowright et al., 2015), our result suggest that following epidemic bursts, the vast majority of infected bats likely develop immunity efficiently and stop excreting pathogens; however, the question of infection maintenance 271 beyond the breeding season has not been addressed. A recent theoretical study, using 272 stochastic epidemiological models with a seasonal birth pulse, suggests that pre-existing 273 immunity is critical for pathogen maintenance in bat populations (Peel et al., 2014). Here, 274 rates of infection at the beginning and end of the breeding season were roughly similar 275 ( 25\%) implying that the bat maternity in fine serves as an ecological "vaccination center", 276 providing potential antibody immunity to large proportions of the population outside of the 277 breeding season. Transmission outside of maternity colonies may thus be maintained by a 278 small fraction of individuals chronically excreting the two pathogens, waning immunity 279 and/or immigration events between colonies (Breed et al., 2011; Field et al., 2011; Sohayati et 280 al., 2011; Peel et al., 2012). As fission-fusion social structures are being increasingly 281 recognized in bats (Kerth et al., 2011), and because the bat species studied herein tends to 282 split into small populations during non-reproductive period, it is possible that a complex 283 meta-population structure will influence seasonal infection dynamics within the community 284 (Plowright et al., 2011). Multidisciplinary research on the interplay between population 285 structures and temporal dynamics in the context of their associated pathogens are important 286 for the prediction of possible emergence events in specific geographic regions (Restif et al., 
2012), such as the western Indian Ocean, where leptospirosis and paramyxoviruses are widely prevalent (Bourhy et al., 2010; Lagadec et al., 2012; Wilkinson et al., 2012; Desvars et al., 2013; Dietrich et al., 2014).

\section{Experimental procedures}

\section{Urine collection}

A colony of the insectivorous bat Mormopterus francoismoutoui was monitored during the 2012-2013 breeding season, in Réunion Island, a tropical oceanic Island located 800km East of Madagascar. The maternity colony is sited in a natural cave of approximately $30 \mathrm{~m}^{3}$ and is occupied by one single bat species, i.e. M. francoismoutoui, from October to May. The maternity colony is mostly composed of adult females at the early stages of the breeding season. A total of eight field outings were carried out from November 2012 to April 2013, a timing that did not allow monitoring the first weeks of the colony formation. We hence conducted additional monitoring in the same cave during the next season in the very early stages of colony formation, with four field outings during October 2013. A non-invasive sampling strategy was implemented in order to avoid direct manipulation of bats thus limiting disturbance of the colony. This strategy is based on the demonstration that infected bats excrete both Leptospira and paramyxovirus in their urine (Baker et al., 2012; Desvars et al., 2013). In addition, urine droplets are spontaneously shed by flying bats during their daily emergence at dusk and could then be collected on plastic films layered on the ground. For each sampling date, 16 plastic film (Saran wrap) -covered cardboard rectangles $(20 \mathrm{x} 15 \mathrm{~cm})$ were placed inside larger plastic trays and positioned at the entrance of the cave following a referenced map. This allowed positioning each tray at the exact same place throughout the whole survey. As shown on the overview of the sampling design provided in Fig. S1 trays were separated by at least 1 meter in order to minimize the possibility of sampling several 
312 urine droplets from a single bat specimen. After the emergence of bats, up to 5 distant (about

$3135 \mathrm{~cm}$ ) urine droplets assumed to correspond to 5 independent bat specimens, were collected

314 from each of the 16 plastic trays and buffered in micro-tubes containing $300 \mu \mathrm{L}$ of Minimum

315 Essential Medium Eagle buffer. Samples were then kept in the field in a cool box filled with 316 ice packs and brought back to the lab where they were immediately stored at $-80^{\circ} \mathrm{C}$ until 317 tested.

\section{Bat colony dynamics}

The colony was visually monitored throughout the whole study in order to estimate the population age structure and timing of parturition. The presence of adults (brown), newborns (pink colored) and juveniles (dark grey) was thus checked by visual inspection at each sampling date (see photos in Fig. S2).

We attempted to characterize changes in bat behavior during the breeding season by monitoring the numbers of bats at emergence. Relative estimates were obtained by counting the number of individual urine droplets landing on the plastic films, as we showed that the number of urine droplets correlates with the number of exiting bats (see details in Fig. S3). Thus, collected figures allowed comparative analysis between sampling field missions.

Video analyses were also used to estimate the absolute size of the exiting population.

330 When lightness and weather conditions permitted, videos of bats leaving the cave were 331 acquired using the sky as a backdrop. Custom MATLAB-encoded algorithms were then used 332 to define a line within the video-images and video frames were segmented based on the 333 contrast of each image (dark bats on a clear sky background). A binary image was then 334 constructed using mask data along the defined line (X-dimension), and for each frame of the 335 video (Y-dimension). Non-touching objects were then counted in the obtained image mask to generate a minimum-number estimate of the number of bats that had left the cave. 


\section{Screening and genetic diversity of Leptospira and Paramyxovirus}

339 Paramyxoviruses are single stranded negative sense RNA viruses, and thus their nucleic acids 340 require reverse transcription before detection via PCR. Therefore, detection was carried out 341 on a single cDNA preparation for both bacterial and viral detection. Using QIAamp Mini spin 342 columns (Qiagen, Valencia, CA), total nucleic acids were extracted following manufacturer's 343 instructions from $140 \mu \mathrm{l}$ of each buffered urine sample. A negative control was added for each 344 extraction run. Following extraction, $10 \mu \mathrm{L}$ of total nucleic acids were submitted to reverse 345 transcription using the Promega cDNA kit (Promega, Madison, USA) with $1.25 \mu \mathrm{L}$ of 346 Hexamers (Promega, Madison, USA), following manufacturer's instructions.

347 Five microliters of cDNA were then used as templates for Leptospira and paramyxovirus 348 detections using previously described detection methods (Smythe et al., 2002; Tong et al., 349 2008). Briefly, the presence of Leptospira was detected using a probe-specific real-time PCR, 350 targeting a fragment of the $16 S r R N A$ gene, specific of all known pathogenic Leptospira 351 species. The threshold cycle $(\mathrm{Ct})$ of positive samples was noted to infer the relative bacterial 352 load and thus the relative intensity of Leptospira excretion. In order to compare $\mathrm{Ct}$ values 353 among different PCR runs, we verified the consistency of the Ct value for the positive control 354 in each run. For positive samples, a partial fragment of the $16 S$ rRNA and $\sec Y$ genes was then 355 amplified, as described in Dietrich et al. (2014), and sequenced (Genoscreen, Lille, France) 356 for subsequent phylogenetic analyses. For the detection of paramyxoviruses, a semi-nested 357 PCR targeting the polymerase-encoding gene was performed as previously described 358 (Wilkinson et al., 2012). After electrophoresis on a 2\% agarose gel stained with GelRed 359 (Biotium Inc.), PCR products of the approximate anticipated size (450-500 bp) were purified 360 using the QIAGEN PCR purification kit, cloned into the pGEMt vector system (Promega), 361 and submitted to direct Sanger sequencing (Genoscreen, Lille, France). DNA sequences of 
362 paramyxoviruses obtained from independent bacterial clones were aligned to generate

363 consensus sequences for each sample in order to correct for the majority of sequencing or 364 PCR-introduced errors. These sequence data have been submitted GenBank database under accession number KJ607934-KJ607957 for Leptospira and KJ748495-KJ748520 for 366 paramyxoviruses

Nucleotide sequences were assembled and edited manually using ChromasLite 2.01

368 (Technelysium Pty Ltd, South Brisbane, Australia), and aligned using CLC Sequence Viewer 6.8 (CLC bio, Aarhus, Denmark) with references sequences including samples from bats and terrestrial small-mammals of the western Indian Ocean Islands (see details in Tables S1 and S2). Phylogenetic trees were constructed using PHYML (Guindon and Gascuel, 2003) under 372 the AIC best-fitted model of evolution (Leptospira: TPM3uf+I and HKY+I for $16 S$ rRNA and 373 secY; Paramyxoviruses: TIM3+I+G) selected by JMODELTEST v.0.1.1 (Posada, 2008), a BIONJ as starting tree and NNI tree search. The robustness of branches was evaluated performing bootstrap analysis with 1000 repetitions.

\section{$377 \quad$ Statistical analyses}

378 We examined changes in the number of urine droplets (as a correlate of the number of bats emerging at dusk) over the breeding season and among the trays by using a Generalized

380 Linear Model (GLM) with a Poisson distribution and a log link function. "Sampling date" and 381 “tray number” were included as explanatory variables.

382 We investigated the dynamic of infections (both mono- and co-infections) by using 383 GLMs with binomial error and a logit link function, with the "sampling date" as an 384 explanatory variable. We aimed to determine whether the presence of one infectious agent 385 was associated with the presence of the alternative infectious agent, adding "infection status" 386 for Leptospira and paramyxoviruses as explanatory variables in models testing mono- 
infections. A G-test was used to test differences between the co-infection rates, as observed or

388 as expected at random. Expected co-infection rates at each sampling date were calculated as 389 the product of the rates of infection with each single pathogen measured at the same date 390 (Nieto and Foley, 2009).

391 The temporal variation in the relative intensity of pathogen excretion in urine was 392 analyzed for Leptospira only, as no accurate quantitative information on viral load could be 393 obtained from the semi-nested PCR used for paramyxovirus detection. We used a GLM with 394 a Gaussian distribution and a logit link function, using the threshold cycle $(\mathrm{Ct})$ as a proxy for 395 the relative intensity of Leptospira excretion and "sampling date", "tray number" and 396 "paramyxovirus infection status" as explanatory variables.

397 All starting models were simplified by backward stepwise elimination of non-significant 398 terms $(p>0.05)$ beginning with interactions, to obtain the minimum adequate model 399 (Crawley, 2007) (see models in Table S3). In models using a binomial error structure, we systematically checked for over dispersion by calculating that the ratio of residual deviance over residual degrees of freedom was <2. Curves were produced using a loess smoother. 402 Evolution of pathogen genetic diversity (proportion of the different lineages over time) was 403 analyzed using a Fisher's exact test. All analyses were carried out using the R software 404 package v.3.0.2.

\section{Acknowledgements}

407 We are grateful to Dominique Bang for kindly giving us access to the bat maternity colony, 408 and to Marina Béral, Lisa Cavalerie, Yann Gomard, Camille Lebarbenchon, Gildas Le 409 Minter, and Julien Mélade for their help in field sampling. We thank Audrey Jaeger and 410 Olivier Flores for advice on statistical analyses. This work was supported by the CPER/ 411 Regional council/European Regional Development Funds ERDF- POCT; Réunion, LeptOI 
$412\left(\mathrm{~N}^{\circ} 32913\right)$, FSOI ( $\left.{ }^{\circ} 31189\right)$ and ParamyxOI ( $\left.{ }^{\circ} 33857\right)$ projects, granted to $\mathrm{PT}, \mathrm{KD}$ and

413 DAW respectively. The postdoctoral fellowships of MD and DAW were funded by "RUN-

414 Emerge: A Regpot European project funded by European Commission under FP7 program".

415 BR received postdoctoral grants from RunEmerge project, from "Fonds de Coopération

416 Régionale" of the Préfecture de La Réunion and from the Dr. Ralph and Marian Falk Medical

417 Research Trust to The Field Museum of Natural History, Chicago. The authors declare no 418 conflict of interest.

\section{References}

Adler, B. and de la Peña Moctezuma, A. (2010) Leptospira and leptospirosis. Vet. Microbiol. 140: 287-96.

Aguilar, H.C. and Lee, B. (2011) Emerging paramyxoviruses: molecular mechanisms and antiviral strategies. Expert Rev. Mol. Med. 13: e6.

Altizer, S., Dobson, A., Hosseini, P., Hudson, P., Pascual, M., and Rohani, P. (2006) Seasonality and the dynamics of infectious diseases. Ecol. Lett. 9: 467-84.

Amengual, B., Bourhy, H., López-Roíg, M., and Serra-Cobo, J. (2007) Temporal dynamics of European bat lyssavirus type 1 and survival of Myotis myotis bats in natural colonies. PLoS One 2: e566.

Baker, K.S., Suu-Ire, R., Barr, J., Hayman, D.T.S., Broder, C.C., Horton, D.L., et al. (2013) Viral antibody dynamics in a chiropteran host. J. Anim. Ecol. 83: 415-428.

Baker, K.S., Todd, S., Marsh, G., Fernandez-Loras, A., Suu-Ire, R., Wood, J.L.N., et al. (2012) Co-circulation of diverse paramyxoviruses in an urban African fruit bat population. J. Gen. Virol. 93: 850-6.

Baker, K.S., Todd, S., Marsh, G.A., Crameri, G., Barr, J., Kamins, A.O., et al. (2013) Novel, potentially zoonotic paramyxoviruses from the African straw-colored fruit bat Eidolon helvum. J. Virol. 87: 1348-58.

Bharti, A.R., Nally, J.E., Ricaldi, J.N., Matthias, M.A., Diaz, M.M., Lovett, M.A., et al. (2003) Leptospirosis : a zoonotic disease of global importance. Lancet Infect. Dis. 3: $757-771$.

Boulinier, T. and Staszewski, V. (2008) Maternal transfer of antibodies: raising immunoecology issues. Trends Ecol. Evol. 23: 282-288. 
Bourhy, P., Collet, L., Clément, S., Huerre, M., Ave, P., Giry, C., et al. (2010) Isolation and characterization of new Leptospira genotypes from patients in Mayotte (Indian Ocean). PLoS Negl. Trop. Dis. 4: e724.

Breed, A.C., Breed, M.F., Meers, J., and Field, H.E. (2011) Evidence of endemic Hendra virus infection in flying-foxes (Pteropus conspicillatus)--implications for disease risk management. PLoS One 6: e28816.

Calisher, C.H., Childs, J.E., Field, H.E., Holmes, K., and Schountz, T. (2006) Bats: important reservoir hosts of emerging viruses. Clin. Microbiol. Rev. 19: 531-45.

Cattadori, I.M., Boag, B., Bjørnstad, O.N., Cornell, S.J., and Hudson, P.J. (2005) Peak shift and epidemiology in a seasonal host-nematode system. Proc. R. Soc. B 272: 1163-1169.

Christe, P., Arlettaz, R., and Vogel, P. (2000) Variation in intensity of a parasitic mite (Spinturnix myoti) in relation to the reproductive cycle and immunocompetence of its bat host (Myotis myotis). Ecol. Lett. 3: 207-212.

Crawley, M.J. (2007) The R Book John Wiley. Chichester, UK.

Desvars, A., Naze, F., Benneveau, A., Cardinale, E., and Michault, A. (2013) Endemicity of leptospirosis in domestic and wild animal species from Reunion Island (Indian Ocean). Epidemiol. Infect. 141: 1154-65.

Dietrich, M., Wilkinson, D. a, Soarimalala, V., Goodman, S.M., Dellagi, K., and Tortosa, P. (2014) Diversification of an emerging pathogen in a biodiversity hotspot: Leptospira in endemic small mammals of Madagascar. Mol. Ecol. 23: 2783-96.

Drexler, J.F., Corman, V.M., Müller, M.A., Maganga, G.D., Vallo, P., Binger, T., et al. (2012) Bats host major mammalian paramyxoviruses. Nat. Commun. 3: 796.

Drexler, J.F., Corman, V.M., Wegner, T., Tateno, A.F., Zerbinati, R.M., Gloza-Rausch, F., et al. (2011) Amplification of emerging viruses in a bat colony. Emerg. Infect. Dis. 17: 449-456.

Epstein, J.H., Baker, M.L., Zambrana-Torrelio, C., Middleton, D., Barr, J.A., Dubovi, E., et al. (2013) Duration of Maternal Antibodies against Canine Distemper Virus and Hendra Virus in Pteropid Bats. PLoS One 8: e67584.

Ferguson, N.M., Galvani, A.P., and Bush, R.M. (2003) Ecological and immunlogical determinants of influenza evolution. Nature 422: 428-433.

Field, H., de Jong, C., Melville, D., Smith, C., Smith, I., Broos, A., et al. (2011) Hendra virus infection dynamics in Australian fruit bats. PLoS One 6: e28678.

George, D.B., Webb, C.T., Farnsworth, M.L., O’Shea, T.J., Bowen, R.A., Smith, D.L., et al. (2011) Host and viral ecology determine bat rabies seasonality and maintenance. Proc. Natl. Acad. Sci. USA 108: 10208-10213. 
Guindon, S. and Gascuel, O. (2003) A Simple, Fast, and Accurate Algorithm to Estimate Large Phylogenies by Maximum Likelihood. Syst. Biol. 52: 696-704.

Hayman, D.T.S., Bowen, R.A., Cryan, P.M., McCracken, G.F., O’Shea, T.J., Peel, A.J., et al. (2013) Ecology of zoonotic infectious diseases in bats: current knowledge and future directions. Zoonoses Public Health 60: 2-21.

Holt, J., Davis, S., and Leirs, H. (2006) A model of Leptospirosis infection in an African rodent to determine risk to humans: seasonal fluctuations and the impact of rodent control. Acta Trop. 99: 218-25.

Hosseini, P.R., Dhondt, A.A., and Dobson, A. (2004) Seasonality and wildlife disease: how seasonal birth, aggregation and variation in immunity affect the dynamics of Mycoplasma gallisepticum in house finches. Proc. R. Soc. B 271: 2569-2577.

Kallio, E.R., Begon, M., Henttonen, H., Koskela, E., Mappes, T., Vaheri, A., and Vapalahti, O. (2010) Hantavirus infections in fluctuating host populations: the role of maternal antibodies. Proc. R. Soc. B 277: 3783-91.

Kerth, G., Perony, N., and Schweitzer, F. (2011) Bats are able to maintain long-term social relationships despite the high fission-fusion dynamics of their groups. Proc. R. Soc. B 278: 2761-7.

Kunz, T.H., de Torrez, B.E., Bauer, D., Lobova, T., and Fleming, T.H. (2011) Ecosystem services provided by bats. Ann. N. Y. Acad. Sci. 1223: 1-38.

Lagadec, E., Gomard, Y., Guernier, V., Dietrich, M., Pascalis, H., Temmam, S., et al. (2012) Pathogenic Leptospira spp. in bats, Madagascar and Union of the Comoros. Emerg. Infect. Dis. 18: 1696-8.

Matthias, M.A., Díaz, M.M., Campos, K.J., Calderon, M., Willig, M.R., Pacheco, V., et al. (2005) Diversity of bat-associated Leptospira in the Peruvian Amazon inferred by bayesian phylogenetic analysis of $16 \mathrm{~S}$ ribosomal DNA sequences. Am. J. Trop. Med. Hyg. 73: 964-74.

Mühldorfer, K., Speck, S., Kurth, A., Lesnik, R., Freuling, C., Müller, T., et al. (2011) Diseases and causes of death in European bats: dynamics in disease susceptibility and infection rates. PLoS One 6: e29773.

Nieto, N.C. and Foley, J.E. (2009) Meta-analysis of coinfection and coexposure with Borrelia burgdorferi and Anaplasma phagocytophilum in humans, domestic animals, wildlife, and Ixodes ricinus-complex ticks. Vector Borne Zoonotic Dis. 9: 93-102.

Pappas, G., Papadimitriou, P., Siozopoulou, V., Christou, L., and Akritidis, N. (2008) The globalization of leptospirosis: worldwide incidence trends. Int. J. Infect. Dis. 12: 351-7.

Pedersen, A.B. and Fenton, A. (2007) Emphasizing the ecology in parasite community ecology. Trends Ecol. Evol. 22: 133-9. 
Peel, A.J., Baker, K.S., Crameri, G., Barr, J.A., Hayman, D.T.S., Wright, E., et al. (2012) Henipavirus neutralising antibodies in an isolated island population of African fruit bats. PLoS One 7: e30346.

Peel, A.J., Pulliam, J.R.C., Luis, A.D., Plowright, R.K., Shea, T.J.O., Hayman, D.T.S., et al. (2014) The effect of seasonal birth pulses on pathogen persistence in wild mammal populations. Proc. R. Soc. B 281: 20132962.

Plowright, R.K., Eby, P., Hudson, P.J., Smith, I.L., Westcott, D., Bryden, W.L., et al. (2015) Ecological dynamics of emerging bat virus spillover. Proc. R. Soc. B 282: 20142124.

Plowright, R.K., Field, H.E., Smith, C., Divljan, A., Palmer, C., Tabor, G., et al. (2008) Reproduction and nutritional stress are risk factors for Hendra virus infection in little red flying foxes (Pteropus scapulatus). Proc. R. Soc. B 275: 861-9.

Plowright, R.K., Foley, P., Field, H.E., Dobson, A.P., Foley, J.E., Eby, P., and Daszak, P. (2011) Urban habituation, ecological connectivity and epidemic dampening: the emergence of Hendra virus from flying foxes (Pteropus spp.). Proc. R. Soc. B 278: $3703-12$.

Posada, D. (2008) jModelTest: phylogenetic model averaging. Mol. Biol. Evol. 25: 12531256.

Pourrut, X., Délicat, A., Rollin, P.E., Ksiazek, T.G., Gonzalez, J.-P., and Leroy, E.M. (2007) Spatial and temporal patterns of Zaire ebolavirus antibody prevalence in the possible reservoir bat species. J. Infect. Dis. 196 Suppl: S176-83.

Restif, O., Hayman, D.T.S., Pulliam, J.R.C., Plowright, R.K., George, D.B., Luis, A.D., et al. (2012) Model-guided fieldwork: practical guidelines for multidisciplinary research on wildlife ecological and epidemiological dynamics. Ecol. Lett. 15: 1083-94.

Sheldon, B.C. and Verhulst, S. (1996) Ecological immunology: costly parasite defences and trade-offs in evolutionary ecology. Trends Ecol. Evol. 11: 317-321.

Smythe, L.D., Smith, I.L., Smith, G.A., Dohnt, M.F., Symonds, M.L., Barnett, L.J., and McKay, D.B. (2002) A quantitative PCR (TaqMan) assay for pathogenic Leptospira spp. BMC Infect. Dis. 2: 13.

Sohayati, A.R., Hassan, L., Sharifah, S.H., Lazarus, K., Zaini, C.M., Epstein, J.H., et al. (2011) Evidence for Nipah virus recrudescence and serological patterns of captive Pteropus vampyrus. Epidem 139: 1570-1579.

Telfer, S., Lambin, X., Birtles, R., Beldomenico, P., Burthe, S., Paterson, S., and Begon, M. (2010) Species interactions in a parasite community drive infection risk in a wildlife population. Science 330: 243-6.

Tong, S., Chern, S.-W.W., Li, Y., Pallansch, M.A., and Anderson, L.J. (2008) Sensitive and broadly reactive reverse transcription-PCR assays to detect novel paramyxoviruses. $J$. Clin. Microbiol. 46: 2652-2658. 
Tulsiani, S.M., Cobbold, R.N., Graham, G.C., Dohnt, M.F., Burns, M., Leung, L.K.-P., et al. (2011) The role of fruit bats in the transmission of pathogenic leptospires in Australia. Ann. Trop. Med. Parasitol. 105: 71-84.

Turmelle, A.S., Allen, L.C., Jackson, F.R., Kunz, T.H., Rupprecht, C.E., and Mccracken, G.F. (2010) Ecology of Rabies Virus Exposure in Colonies of Brazilian Free-Tailed Bats (Tadarida brasiliensis) at Natural and Man-Made Roosts in Texas. Vector Borne Zoonotic Dis. 10: 165-175.

Wibbelt, G., Moore, M.S., Schountz, T., and Voigt, C.C. (2010) Emerging diseases in Chiroptera : why bats? Biol. Lett. 6: 438-440.

Wilkinson, D.A., Mélade, J., Dietrich, M., Ramasindrazana, B., Soarimalala, V., Lagadec, E., et al. (2014) Major paramyxovirus exchange between introduced and endemic small mammals in the southwestern Indian Ocean region. J. Virol. 88: 8268-8277.

Wilkinson, D.A., Temmam, S., Lebarbenchon, C., Lagadec, E., Chotte, J., Guillebaud, J., et al. (2012) Identification of novel paramyxoviruses in insectivorous bats of the Southwest Indian Ocean. Virus Res. 170: 159-63. 
568 Fig. 1. Seasonal dynamics of $(\mathrm{a}, \mathrm{b})$ the bat population and (c, d) Leptospira and 569 paramyxovirus infections in the maternity colony of $M$. francoismoutoui. (a) Estimated 570 presence of adults, newborns and juveniles within the colony (black rectangles). (b) 571 Emergence of bats at dusk. The continuous line represents the number of urine droplets lying 572 along the main flight-path of bats exiting the cave at dusk, and the shaded area the standard 573 deviation. (c) Leptospira and paramyxovirus infection rates. The continuous lines represent 574 the proportion of PCR-positive samples for Leptospira (green) and paramyxovirus (purple), 575 and the shaded area the $95 \%$ CI. (d) Intensity of Leptospira excretion. The dashed line 576 represents the mean $\mathrm{Ct}$ values for positive Leptospira samples (represented in its negative 577 form) and the shaded area the standard deviation. In each panel, the x-axis depicts the 578 seasonal time scale in month and the white dots correspond to sampling dates.

Fig. 2. (a) Proportion of mono- and co-infections among infected individuals and (b) seasonal dynamics of co-infections in the $M$. francoismoutoui maternity colony. (a) White bars 582 represent mono-infected individuals and grey bars individuals with co-infection. Error bars 583 represent $95 \%$ confidence intervals. (b) The continuous line represents the observed rate of 584 co-infection, while the dashed line corresponds to the expected probability at random. 95\% CI 585 are represented by shaded areas. The x-axis depicts the seasonal time scale in month and the 586 white dots correspond to sampling dates.

588 Fig. 3. Phylogenetic relationships of (a) Leptospira and (b) paramyxovirus detected in the maternity colony of M. francoismoutoui. Samples from this study are represented in green for 590 Leptospira and purple for paramyxovirus and are coded with sample ID and date (month-day591 year). Reference sequence labels refer to the host species and geographic location. Genbank 
592 accession numbers are indicated in parentheses. Bootstrap values higher than $80 \%$ are 593 represented by a dark circle.

594 